\title{
Rezidivierende Hypoglykämien
}

\section{Mit dem Fastentest zur Diagnose}

\author{
Rezidivierende Unterzuckerungen plagten eine 32-jährige Patientin schon seit mehreren Jahren. \\ Bei der Abklärung erwies sich der Fastentest als diagnostisch wegweisend.
}

_ Bei der 32 Jahre alten Frau traten sei acht Jahren rezidivierende Hypoglykämien auf, wobei ein Abfall der Blutzuckerwerte bis auf $30 \mathrm{mg} / \mathrm{dl}$ dokumentiert wurde. Diese Episoden gingen mit Vigilanzminderung, Schwäche, Schweißausbrüchen, Zittern und Schwindel einher. Die Anfälle von Unterzuckerung traten gehäuft nachts oder vor dem Frühstück auf.

Die klinische Untersuchung ist jetzt - abgesehen von einem BMI von $30 \mathrm{~kg} / \mathrm{m}^{2}$ - unauffällig.

\section{Zahlreiche Differenzialdiagnosen kommen in Betracht}

„Bei der differenzialdiagnostischen Abklärung einer Hypoglykämie muss vieles diskutiert werden“, erklärte Dr. Dilek Sadet von der Medizinischen Universitätsklinik in Frankfurt a. M. Dabei sollte man sich auch vom klinischen Bild,

\section{Hungerversuch}

Der Hungerversuch bzw. 72-Stunden-Fastentest dient dazu, bei wiederholten ungeklärten Hypogläkmien den Verdacht auf das Vorliegen eines Insulinoms auszuschließen oder zu erhärten. Der Patient nimmt bei reichlicher Flüssigkeitszufuhr 72 Stunden lang keine Nahrung zu sich. Alle vier Stunden werden Blutzucker, Insulin und C-Peptid gemessen, bei Blutzuckerwerten unter $60 \mathrm{mg} / \mathrm{dl}$ erfolgen die Messungen stündlich. Fällt der Zuckerwert zweimal unter $40 \mathrm{mg} / \mathrm{dl}$, wird der Versuch abgebrochen, ebenso bei Werten unter $50 \mathrm{mg} / \mathrm{dl}$, falls Symptome der Hypoglykämie auftreten. also dem Allgemeinzustand des Patienten leiten lassen. Erscheint er gut genährt und gesund, sollte vorrangig an Medikamente bzw. eine Hypoglycaemia factitia oder ein Insulinom gedacht werden. Bei Patienten mit einem schlechten Allgemeinzustand kommen differenzialdiagnostisch auch eine Unterfunktion der Hypophyse, ein Morbus Addison, eine Niereninsuffizienz, eine Anorexie oder eine Glykogenspeicherkrankheit in Betracht.

\section{Verdachtsdiagnose bestätigt sich}

Da sich die Patientin in einem guten Allgemeinzustand befindet und nicht an Gewicht abgenommen hat, wird primär an ein Insulinom gedacht.

Der Goldstandard zum Nachweis eines solchen ist der 72-Stunden-Fastentest mit der Whipple's Trias: niedriger Blutzucker mit Unterzuckerungszeichen bei erhöhten Werten von Insulin und CPeptid sowie rasche Beseitigung der Symptome nach Kohlenhydrataufnahme.
In der Tat führt der Fastentest bei der Patientin zu einem Blutzuckerabfall auf $31 \mathrm{mg} / \mathrm{dl}$ mit entsprechender Symptomatik. Somit ist die Diagnose „Insulinom“ gesichert.

\section{Lokalisationsdiagnostik mit CT oder MRT}

Da die transabdominelle Sonografie keine ausreichend hohe Sensitivität bietet, sollte zur Lokalisationsdiagnostik ein CT oder MRT durchgeführt werden. Eine Angiografie kann aber ergänzend zur rein morphologischen Darstellung, insbesondere bei atypischen Insulinomen, durchaus hilfreich sein.

Therapie der Wahl ist immer die Operation. Nur bei inoperablem Patienten kann eine symptomatische Therapie mit Diazoxid durchgeführt werden. Selten ist das Insulinom Teil einer multiplen endokrinen Neoplasie (MEN); dann finden sich weitere endokrin-aktive Tumoren im Bereich der Nebenschilddrüsen, des Duodenums (Gastrinom), der Hypophyse (Somatostatinom) oder des Pankreas (Glukagonom).

Dr. med. Peter Stiefelhagen

- Quelle: Fallvorstellung von Dr. Dilek Sadet, Frankfurt a.M., im Rahmen der Herbsttagung der DDG, 11. November 2016 in Nürnberg

\section{Fabula docet:}

Bei rezidivierenden Hypoglykämien sollte nach Ausschluss einer medikamentösen Ursache immer an ein Isulinom gedacht werden. Der Goldstandard für die Diagnosestellung ist der 72-Stunden-Fastentest mit der Whipple's Trias. 